\title{
Contrast-Enhanced Ultrasound (CEUS) in Non-Traumatic Abdominal Emergencies
}

\section{(๑)(8) $\odot$}

Authors

Diletta Cozzi ${ }^{1}$, Simone Agostini ${ }^{1}$, Elena Bertelli ${ }^{1}$, Michele Galluzzo ${ }^{2}$, Emanuela Papa ${ }^{3}$, Germano Scevola $^{3}$ (D), Margherita Trinci², Vittorio Miele ${ }^{1}$

\author{
Affiliations \\ 1 Azienda Ospedaliero Universitaria Careggi, Emergency \\ Radiology, Firenze, Italy \\ 2 Azienda Ospedaliera San Camillo Forlanini, Emergency \\ Radiology, Roma, Italy \\ 3 Ospedale Sandro Pertini, Interventional Radiology, Roma, \\ Italy
}

Key words

ultrasound; $C T$, acute, abdomen

received $\quad 03.08 .2020$

accepted after revision $\quad 01.05 .2021$

Bibliography

Ultrasound Int Open 2021; 7: E76-E86

DOI 10.1055/a-1347-5875

ISSN 2199-7152

(c) 2021. The Author(s).

This is an open access article published by Thieme under the terms of the Creative Commons Attribution-NonDerivative-NonCommercial-License, permitting copying and reproduction so long as the original work is given appropriate credit. Contents may not be used for commecial purposes, or adapted, remixed, transformed or built upon. (https://creativecommons.org/ licenses/by-nc-nd/4.0/)

Georg Thieme Verlag KG, Rüdigerstraße 14,

70469 Stuttgart, Germany

\author{
Correspondence \\ Diletta Cozzi \\ Emergency Radiology \\ Azienda Ospedaliero Universitaria Careggi \\ Largo Brambilla 3 \\ 50139 Firenze \\ Italy \\ dilettacozzi@gmail.com
}

\section{ABSTRACT}

Conventional ultrasound imaging (US) is the first-line investigation in acute non-traumatic abdominal emergencies, but sometimes it needs further examinations, such as computed tomography $(\mathrm{CT})$, to reach a certain diagnosis. Contrast-enhanced ultrasound (CEUS), through injection of contrast medium, may provide the radiologist with additional information that could not be investigated with baseline US. It could help reach a diagnosis and rapidly determine the proper therapy in an emergency setting. The purpose of this review is to explain and illustrate the various possibilities and limitations of CEUS in acute non-traumatic abdominal diseases, in particular acute inflammation, parenchymal infarcts, and hemorrhages.

\section{Manuscript}

In an emergency setting, ultrasound (US) imaging is the first-line approach, and it has a fundamental role in detecting and diagnosing various acute abdominal pathologies [1-3]. The many applications of US imaging in the study of abdominal diseases make US examination a versatile modality. Nevertheless, standard US imaging has several limitations in characterizing anatomic structures and vascularized lesions. Contrast-enhanced ultrasound (CEUS) is a technique that uses specific contrast agents that can improve the characterization of anatomical structures through the visualization of small vessels and microcirculation [4-7].

CEUS contrast agents are microbubbles made of a gas core and a stabilized biological shell. The second generation of sonography contrast media is a solution prepared very quickly and it is imme- diately administrable. CEUS has the benefit of a high temporal resolution and the operator can detect the contrast transit in the arterial, portal-venous, and late phase. The arterial phase starts $10 \mathrm{sec}$ after injection and lasts $25-30 \mathrm{sec}$. The portal-venous phase starts immediately after the arterial one. Furthermore, these contrast agents have a short half-life, are excreted rapidly from the lungs, and are very well tolerated. In fact, they can be injected into patients with renal insufficiency, hypotension, shock, without further preliminary laboratory tests, and are also indicated in critical patients $[4,5,8]$. CEUS contrast media are safe with a very low incidence of side effects. There are no cardio-, nephro- or hepato-toxicities and life-threatening anaphylactic reactions in abdominal applications have been reported with a rate of $0.001 \%$ in the study of O'Connor et al. [9]. In Europe, CEUS has only been approved for a 
limited number of non-traumatic abdominal pathologies in adults, focusing especially on liver disease. Various studies have shown it to be an excellent imaging technique also for non-hepatic applications. A fundamental practical approach is described in the EFSUMB Guidelines [10-12]. CEUS application in children is currently still "off-label" except for a few indications including vesico-uereteral reflux, but it could be a very useful method in the non-ionizing imaging of young patients [6, 13-17].

Today, in the emergency setting, CEUS is used predominantly in the study of blunt or penetrating traumatic lesions, especially for the diagnosis and follow-up of the solid parenchymal organ (socalled "non-operative management") [8,18-22]. Often CEUS is used after a baseline US examination, in an attempt to avoid radiation exposure a computed tomography (CT) examination, especially in hemodynamically stable minor trauma. Furthermore, CEUS is a valid method that can help the radiologist make rapid decisions in case of an unstable major trauma that cannot be moved to the radiology emergency department and needs immediate surgical treatment $[8,19,20,23,24]$.

The experience with CEUS in abdominal non-traumatic pathology is still limited. In fact, the purpose of this article is to review the possibilities and limitations of CEUS in the acute abdominal setting, focusing on various scenarios (inflammation, ischemia, and hemorrhage) and their related radiological images.

\section{Intra-abdominal hemorrhage}

Intra-abdominal hemorrhage can be caused by spontaneous bleeding, for example during anticoagulant therapy, spontaneous rup- ture of a solid mass, a hepatocellular carcinoma, an aortic aneurysm, or a hemorrhage within cystic lesions ( - Fig. 1) [5, 25]. It can be secondary also to diagnostic procedures or therapies such as biopsy or femoral artery puncture in interventional maneuvers. Baseline US can detect the collection of bloody in the peritoneal or extraperitoneal space. Performing CEUS exam helps to recognize the real site of bleeding, through the direct visualization of the contrast leakage (similar to in angiography or during CT exam after contrast injection) [26]. On CEUS, the operator sees active contrast extravasation directly from a vessel (in a continuous or pulsatile form), like in the case of a spontaneous rupture of an abdominal vessel. The extravasation is seen as enhancing blood passing through the arterial wall defect and adjacent pooling [27]. CEUS may have difficulties in differentiating active free bleeding from a pseudoaneurysm. In this case, the differential diagnosis must be made through angiography or CT ( $>$ Fig. 2) [26, 28-30]. In the case of a ruptured abdominal mass, CEUS examination can detect the contrast bleeding within the tumor or into the adjacent hematoma ( $\triangleright$ Fig. 3) [31]. Also, in the case of patients with a complicated abdominal cystic mass or a vesical hemorrhage, CEUS can depict the bleeding site and differentiate it from active contrast extravasation or endoluminal clots (for example, in the case of massive hematuria). However, CEUS exam cannot depict small, deep, and multiple hemorrhages, especially those located outside the scanned area. In these cases, $\mathrm{CT}$ with contrast media injection is the main radiological examination to be performed, because of its panoramic and direct visualization of the exact bleeding vessel [32]. Moreover, it has to be remembered that clinical evaluation is fundamental. For example, with both B-mode and CEUS it is not possible to differen-

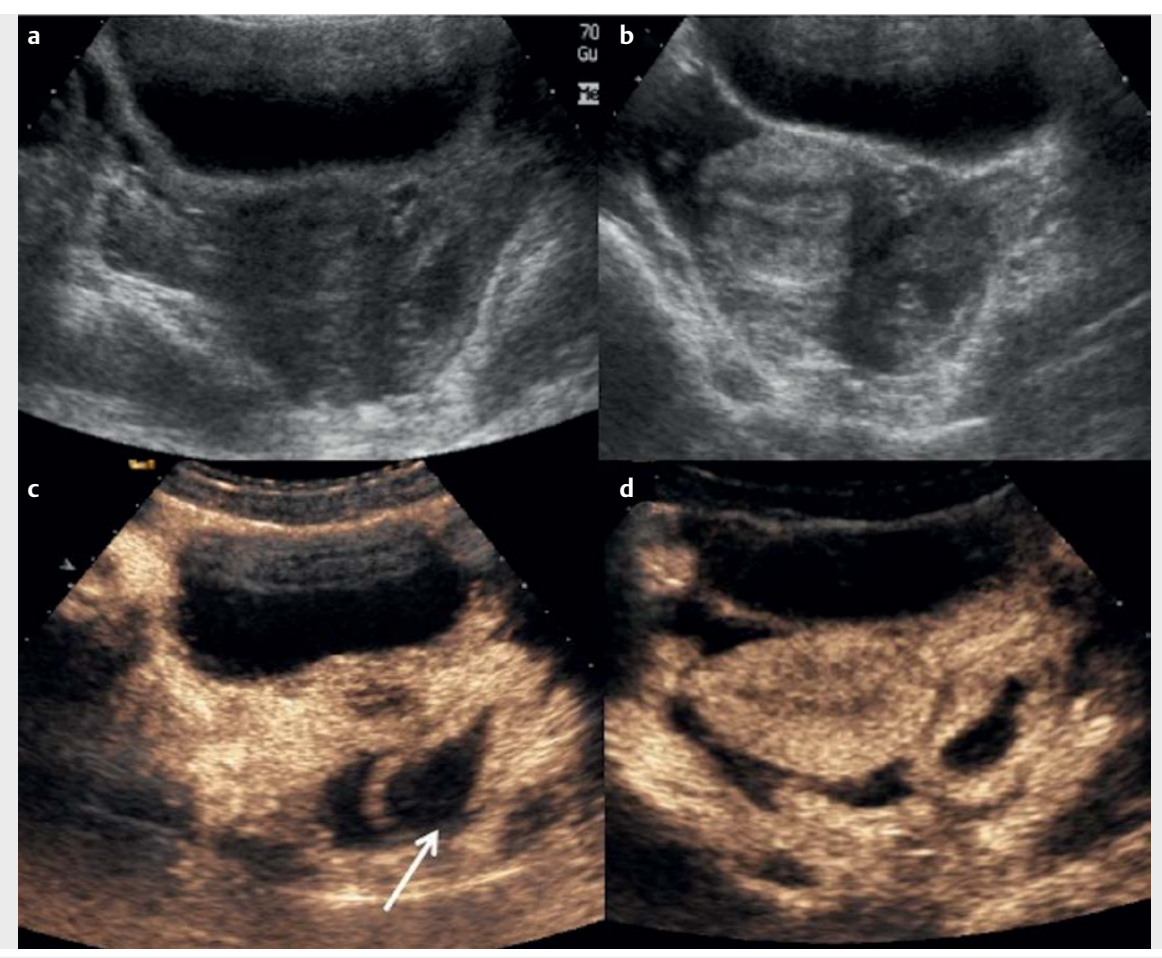

- Fig. 1 Hemorrhagic corpus luteum. A young girl 14 y.o. with pelvic pain arrives at the emergency department and the US examination shows the presence of an enlarged left ovary compared to the contralateral. The CEUS exam shows the presence of anechoic (internal) cystic formation with an enhancing wall and an interruption of the peripheral profile (white arrow), findings compatible with rupture of the corpus luteum. It is also associated with free fluid in the pelvis. 
tiate between a spontaneous or traumatic splenic rupture, because the imaging findings are the same [33, 34].

\section{Parenchymal ischemia and infarction}

In the case of parenchymal ischemia and infarction, CEUS can easily demonstrate the non-vascularized area, especially in solid organs like the spleen, liver, and kidney (that are also effectively examined by ultrasonography examination) [33, 34]. Suspected hypoechoic areas due to hypoperfusion already detected on baseline US can be confirmed with CEUS, which effectively shows the presence of microcirculation alterations. In fact, the alterations in perfusion after the administration of the ultrasound contrast medium appear as clearly visible hypoperfused areas due to the high intrinsic lesion-to-parenchyma contrast of CEUS [5]. In these cases, CEUS can be used instead of CT to reach a correct diagnosis and evaluate the extent of the damage. It is a valid method to perform noninvasive follow-up especially if the infarction is treated conservatively. A recent review by Tedesco et al. shows the diagnostic approach of CEUS in infarctions of the kidney, spleen and pancreas. This affirms that CEUS can replace CT in specific situations [10, 35, 36]. Furthermore, the study by Setola et al. describes the important role of CEUS in confirming suspected renal infarction on baseline US ( [37]. The same CEUS semeiotics can be seen in testicular or ovarian torsion and infarction, torsion of the spermatic cord and epididymis, as described in the study by Badea et al. ( $\mathbf{F i g . ~ 5 , ~ 6 ) ~}$ $[12,38-41]$. An advantage of CEUS in the pediatric population is the lack of radiation exposure. CEUS has also been reported useful in evaluating intestinal ischemia and small bowel infarction [42, 43]. Hata et al. reported a sensitivity of $85-100 \%$ and a specificity of $98-100 \%$ in diagnosing intestinal infarction with CEUS, from various etiologies [44].

\section{Abdominal inflammation}

Similar to ischemia and infarction, in inflammation and phlogosis, there is a higher lesion-to-parenchyma contrast ratio in CEUS examination where the hyperemia corresponds to increased parenchymal microcirculation. CEUS in inflammatory disease has a key role in differentiating between an acute phlogosis and a real abscess or phlegmon or inflammation from a gangrenous evolution $[10,45,46]$. This principle is applied to any acute abdominal inflammatory disease from cholecystitis to appendicitis, pancreatitis, pyelonephritis, or diverticulitis. In particular, in acute cholecystitis, CEUS can very easily detect the evident mural enhancement and any possible gangrenous evolution. In the last case, the lack of mural enhancement is a sign of perforation indicating the need for immediate surgical treatment ( Fig. 7) [47, 48]. Similarly, in acute appendicitis, CEUS improves the detection rate of the disease achieving $98 \%$ accuracy and $100 \%$ sensitivity in the diagnosis, as reported by Incesu et al. [49]. This method can be used especially in the pediatric population after baseline US, avoiding a CT examination. CEUS has been shown to have sufficient accuracy to determine complications (such as perforated or phlegmonous

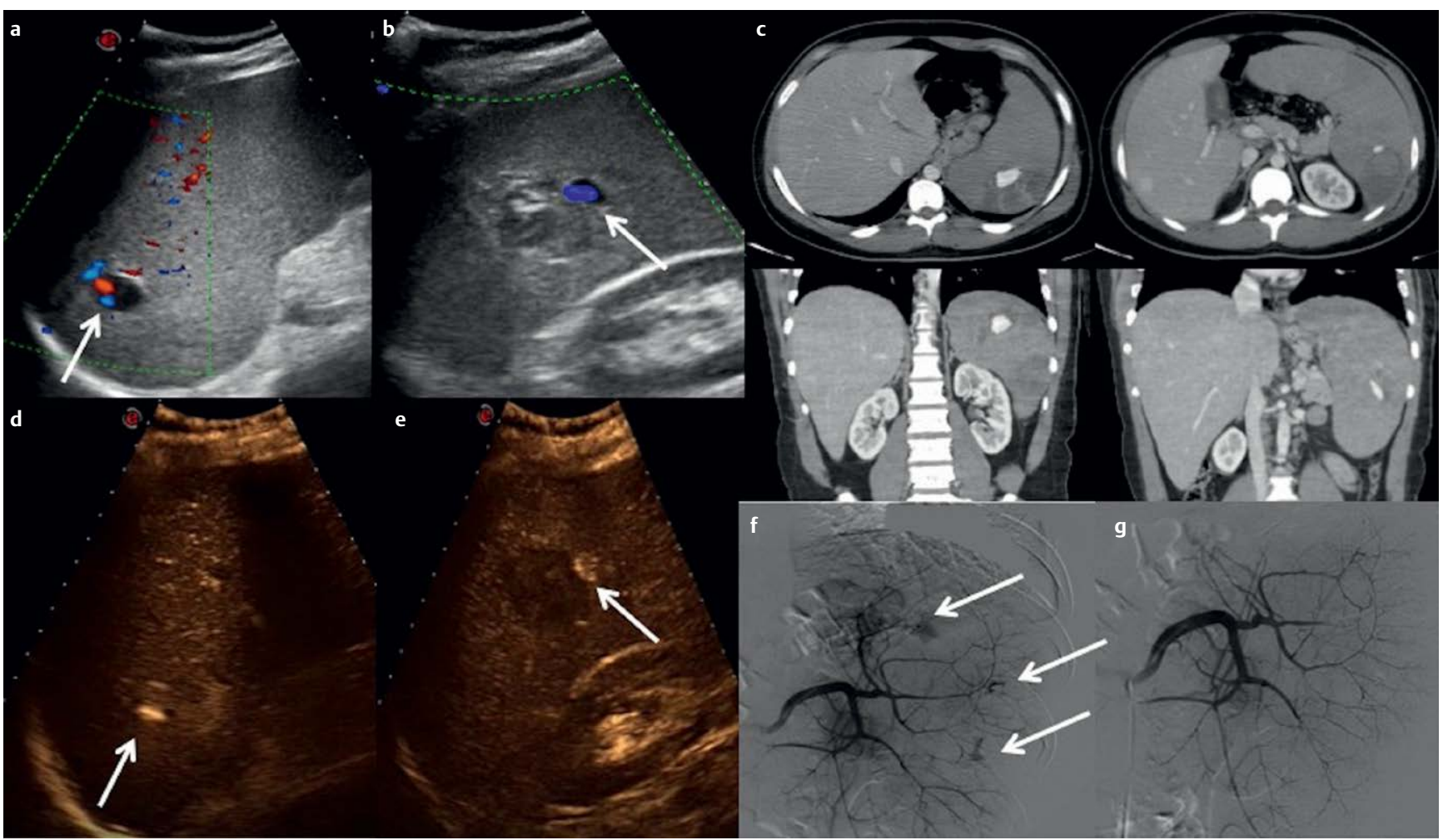

Fig. 2 Splenic pseudoaneurysm. Young male patient 18 y.o. arrives at the emergency department with upper-left quadrant pain. Baseline US (arrows in a-b) demonstrates the presence of at least two parenchymal anechogenic lesions with peripheral vascular signal at Doppler exam. CEUS (d-e) and CT (C) confirm the presence of two vascular lesions within the splenic parenchyma referable to pseudoaneurysm. The diagnosis of certainty and treatment were then made with angiography and embolization of pseudoaneurysms (f). 


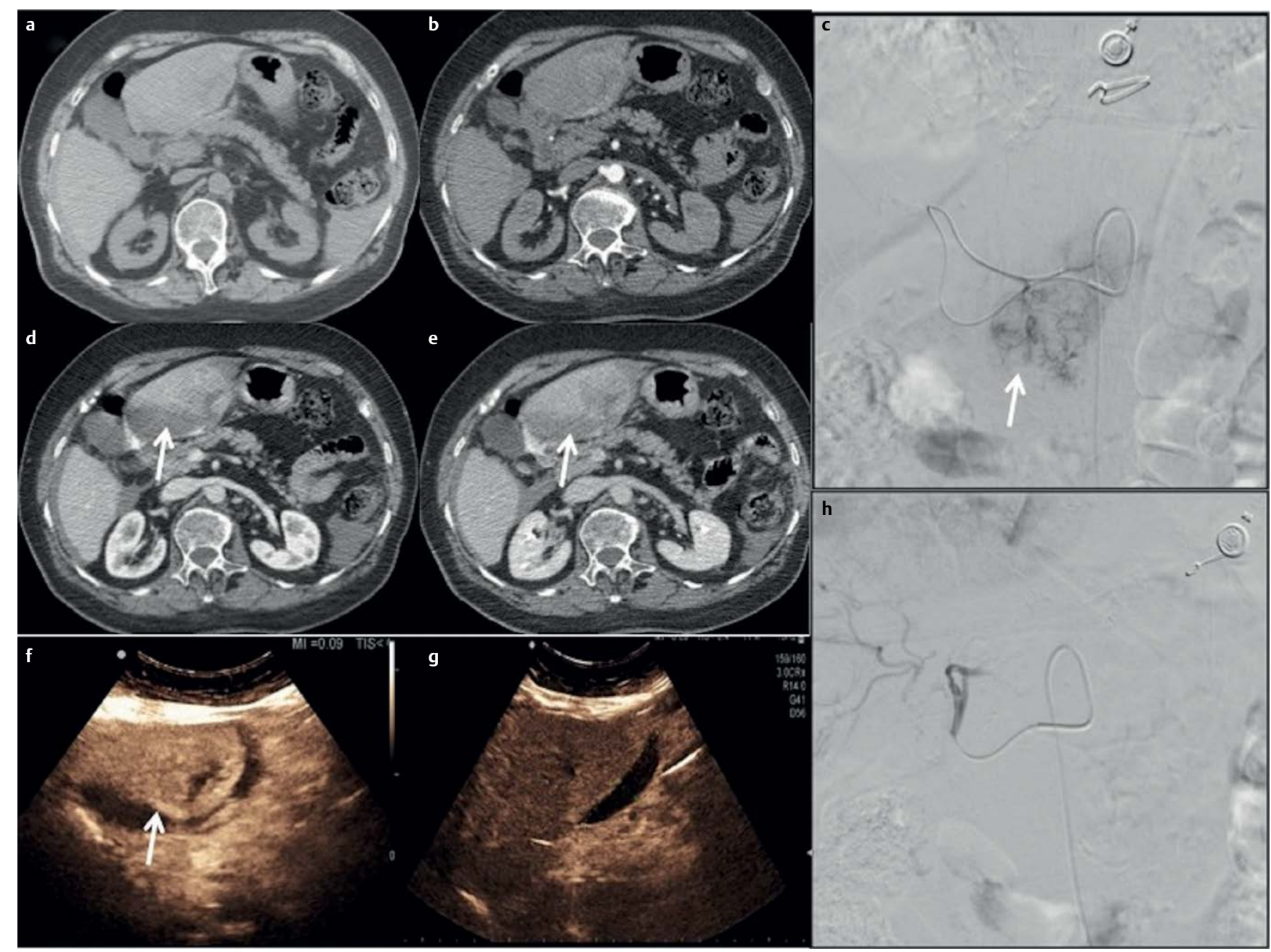

- Fig. 3 Spontaneous HCC rupture. Case of spontaneous bleeding from an unknown liver lesion in a patient without additional comorbidities, which turned out to be hepatocellular carcinoma. CT with contrast medium (a-b-d-e) highlights the bleeding lesion in the left lobe, then confirmed by the CEUS examination (arrow in $\mathbf{f}$ ) where there is a subcapsular arterial blush indicative of bleeding in progress, which also extends into the adjacent peritoneal cavity. Angiography confirms the presence of active bleeding (c), embolized and no longer evident in angiographic (h) and CEUS controls $(\mathbf{g})$.

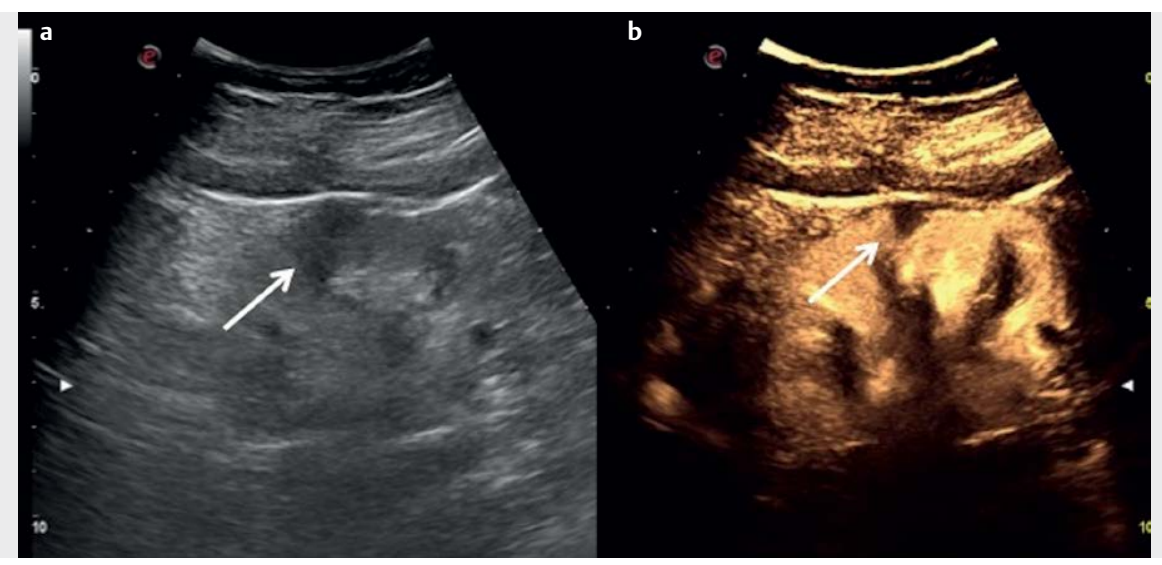

- Fig. 4 Kidney infarct. Right cortical mesorenal infarction with triangular morphology, poorly visualized at baseline US (a) but without enhancement at CEUS (white arrow - b) compared to the remaining vascularized parenchyma. 


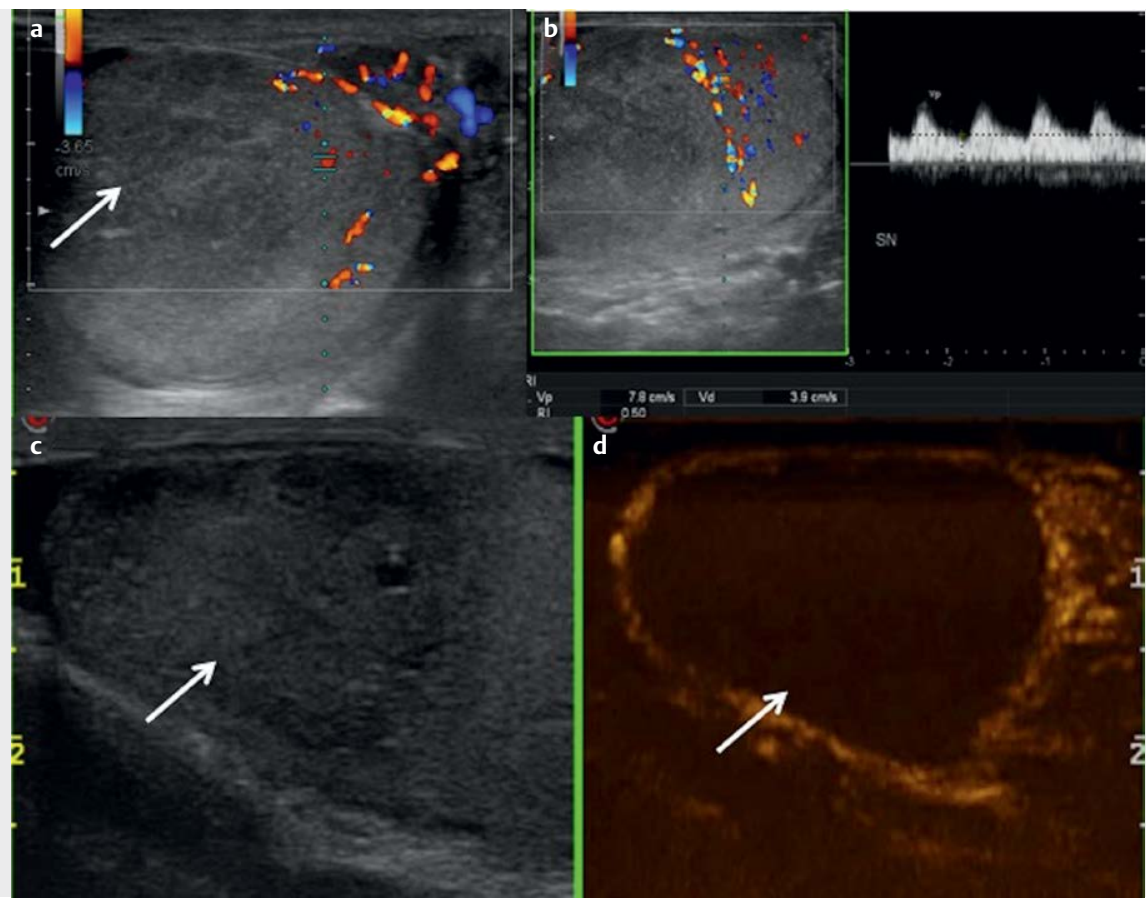

- Fig. 5 Acute testicular infarct. Segmental ischemia of a testicle visualized as a diffusely hypoechoic area with poor Doppler representation (white arrow in $\mathbf{a}-\mathbf{b}-\mathbf{c}$ ). The infarcted part of the testicle is not vascularized or enhanced on CEUS (d).

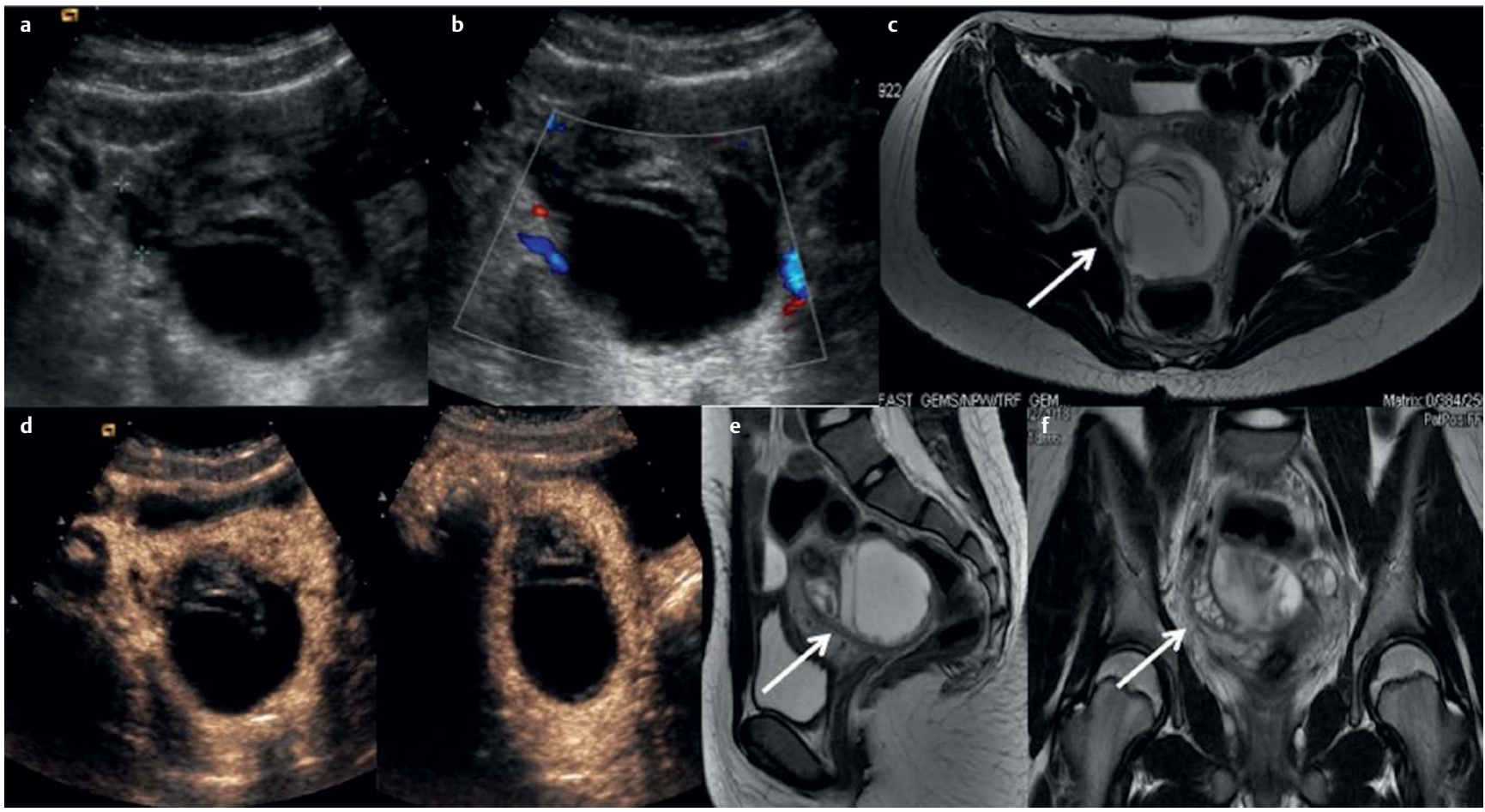

- Fig. 6 Acute ovarian torsion. Young girl 10 y.o. comes to the emergency department complaining of constant pain during defecation. The first US examination (a-b) demonstrates the presence of a coarse cystic mass within the right ovary, with slightly thickened walls with poor peripheral vascularization. CEUS (d) demonstrates the presence of a cystic lesion with poorly enhanced walls and with solid endoluminal parietal projections. The patient completed the diagnostic procedure with MRI (axial c, sagittal $\mathbf{e}$ and coronal $\mathbf{f}$ T2-weighted scans) which confirmed the presence of the adnexal lesion (arrow). Note the perfect agreement between the ultrasound images, CEUS, and MRI. The lesion turned out to be a cystic teratoma. 


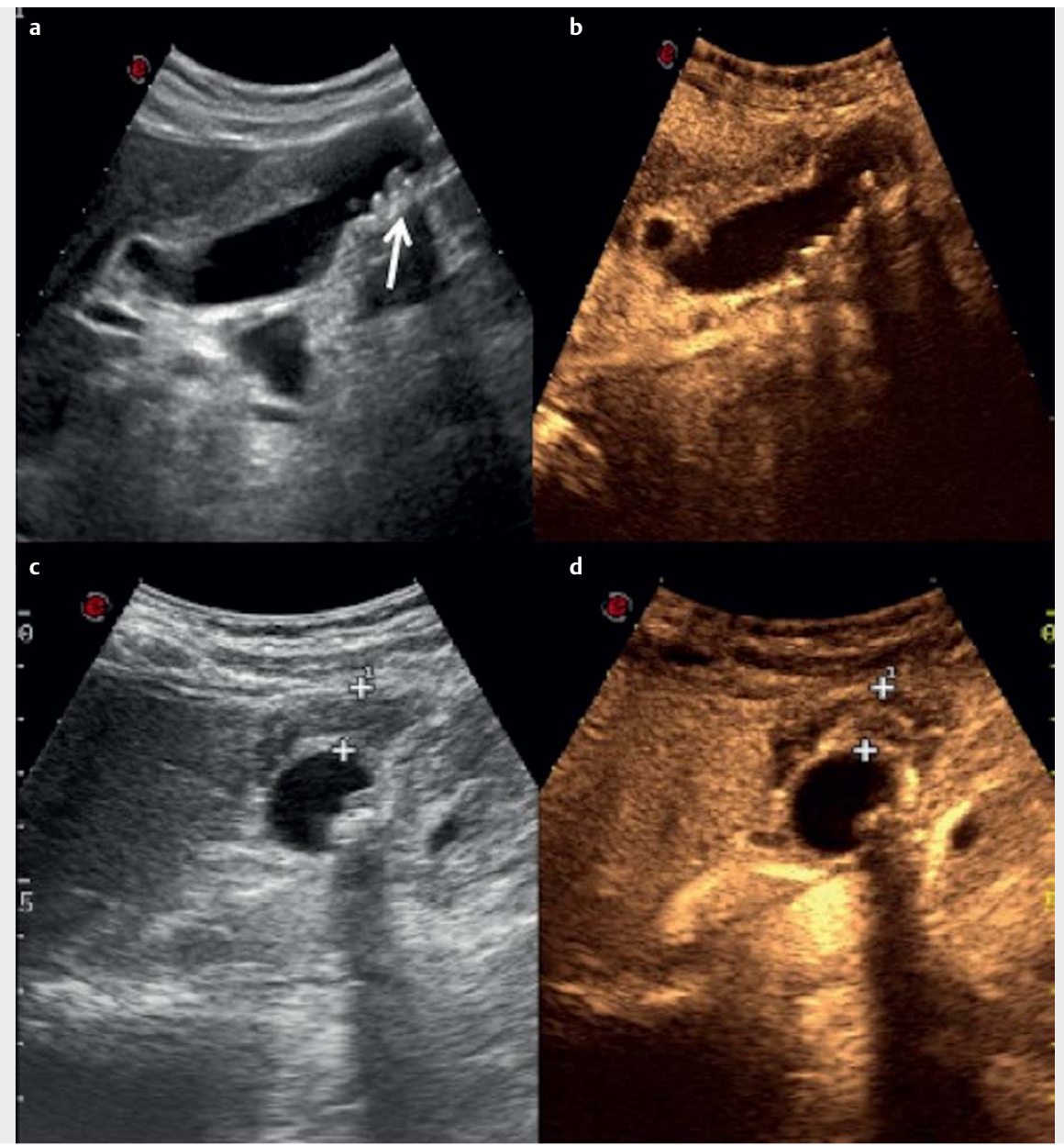

- Fig. 7 Acute complicated cholecystitis. On the baseline US images (a and $\mathbf{c}$ ) there are recognizable signs of cholecystitis with a gallbladder filled with sludge and microcalculi (arrow). CEUS highlights the evident enhancement of the gallbladder wall, which appears very thickened (c and $\mathbf{d}$ ), without showing signs of complication such as an abscess or perforation.

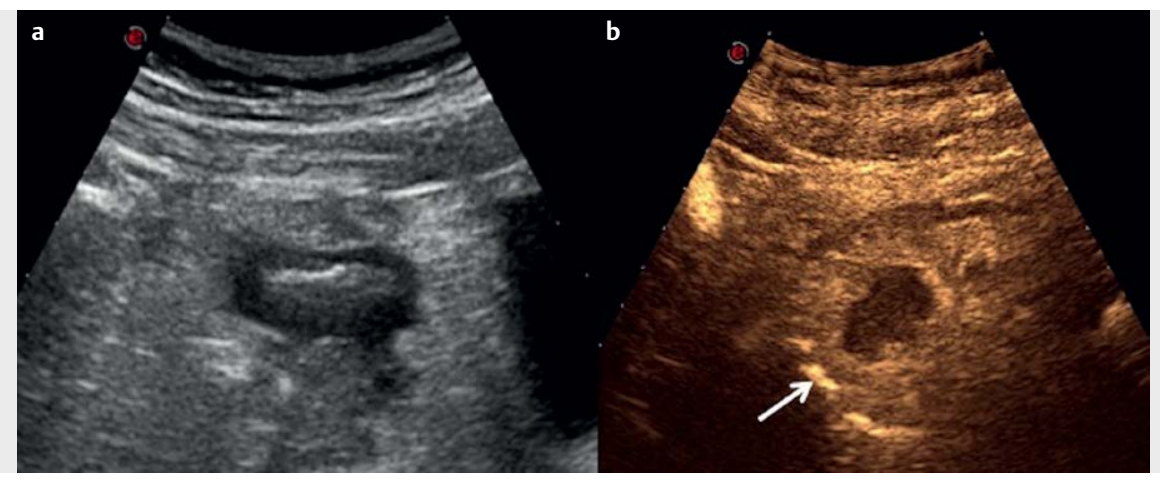

-Fig. 8 Acute diverticulitis. In a, the intestinal wall is visualized an axial scan, highlighting a hypoechoic exophytic lesion suggestive of a diverticulum. After administration of US contrast medium $\mathbf{b}$, the evident enhancement of the inflamed intestinal wall, in the arterial phase (arrow).

and gangrenous appendicitis) [13,49]. Another cause of abdominal pain that can mimic appendicitis is epiploic appendagitis. Also, in this case, CEUS may help the diagnosis by highlighting rounded enhancement in the abdominal fatty tissue within a necrotic center, which is a typical finding in the disease [50]. In the case of acute pancreatitis, CEUS could be used to define the area of higher vascularization within the parenchyma. This technique is not used in routine daily work, first of all, because patients with acute pancre- 


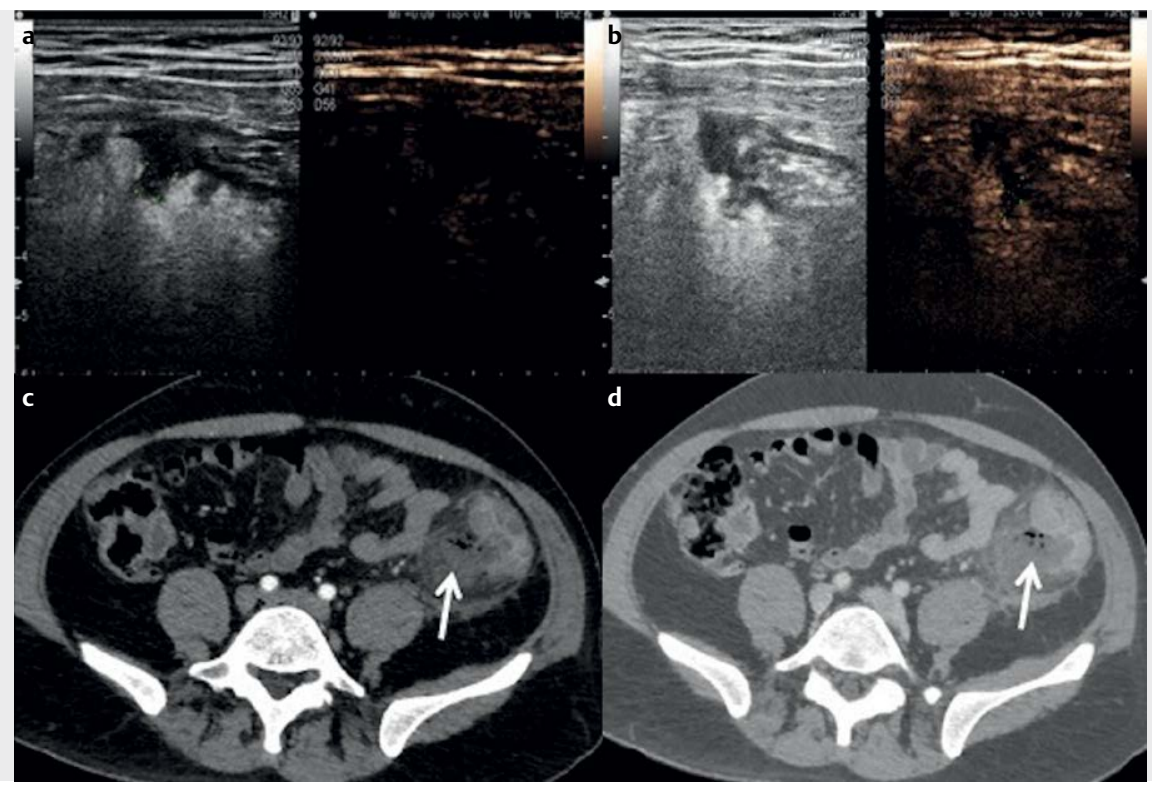

- Fig. 9 Abscess in diverticulitis. Pain in the left iliac fossa in a man without further comorbidity. In the clinical suspicion of an abscess, a CEUS (a-b) examination was performed directly: it shows a nonhomogeneous constantly anechogenic fluid collection with marked peripheral arterial enhancement, typical of an abscess. The finding was confirmed by CT (c-d) which also makes small air bubbles stand out better, in the context of a peri-diverticular abscess.

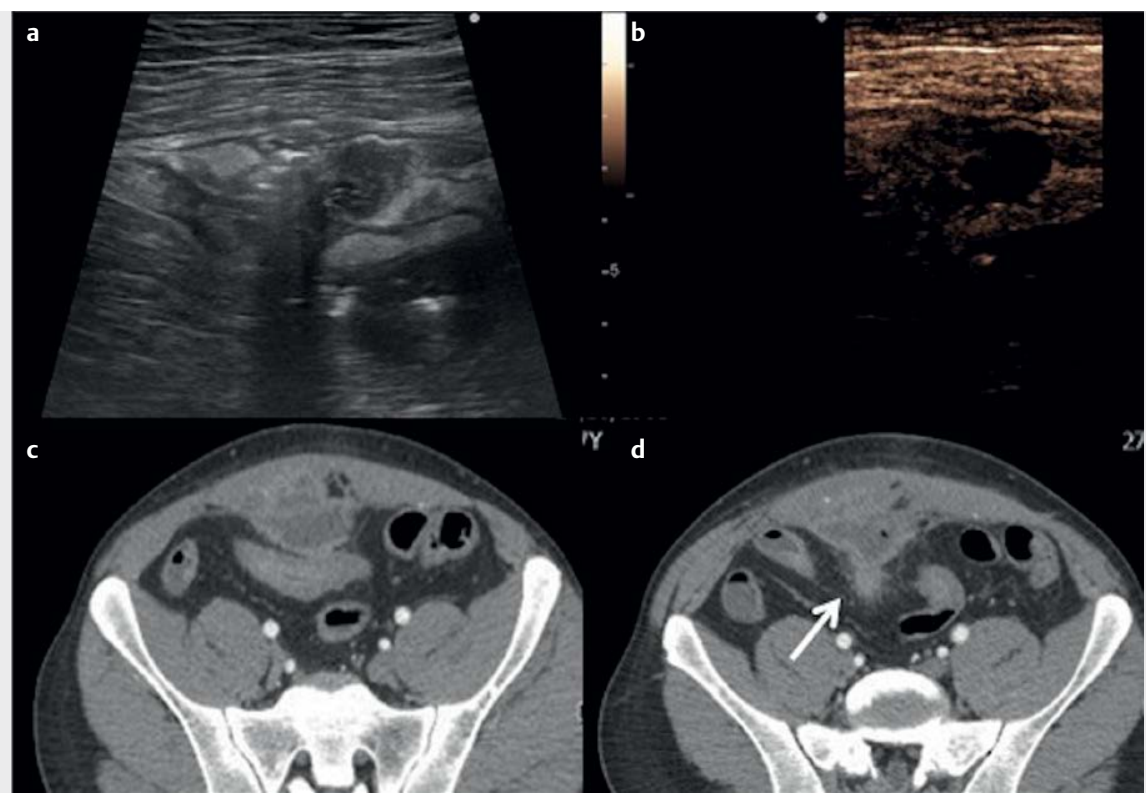

Fig. 10 Abscess in Crohn's disease. Similarly to > Fig. 9, this is a case of a 37-year-old male patient with Crohn's disease with an intestinal abscess tightly attached to the abdominal wall (arrow).

atitis have pain and it is not easy to examine the whole pancreas on US $[10,51]$. In this case, CT exam performed in the emergency department is the gold standard and helps to grade the process [52].

In the case of acute gastrointestinal inflammation caused by diverticulitis, appendicitis, or inflammatory bowel disease (IBD - like Crohn disease), CEUS can improve the detection of their complications, such as abscess and phlegmon ( Fig. 8, 9) [53-55]. Abscess appears as a non-enhancing rounded lesion with hyperemic borders, while phlegmons are mass-like with a non-homogeneous pat- tern of enhancement ( $\triangleright$ Fig. 10) $[53,56]$. IBD is a lifelong chronic disease and patients are often young. Currently, US and CEUS are similar to $\mathrm{CT}$ and magnetic resonance imaging (MRI) in detecting active disease and follow-up of intestinal lesions $[57,58]$. Detection and evaluation of vascular flow in the bowel wall is an important factor in the diagnosis and management of IBD. On baseline US, it may de difficult to distinguish the necrotic liquefied center of an abscess, but after contrast injection, the presence of nonenhanced necrotic central nucleus and a peripheral enhanced wall 

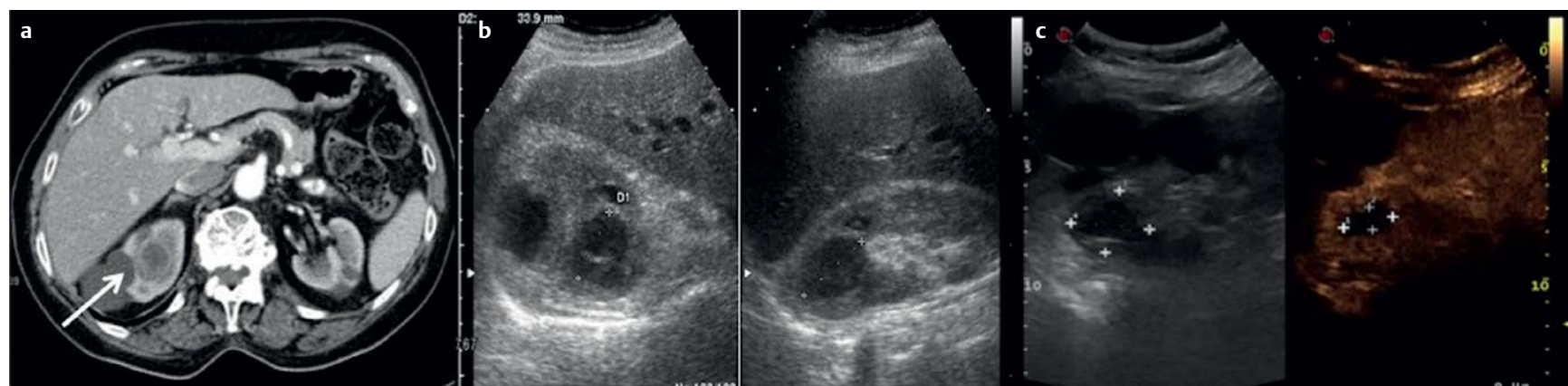

- Fig. 11 Renal abscess. CT a and US (b-c) signs of a renal abscess in the upper right pole in an elderly patient (arrow). The CEUS study (c) confirms the presence of an abscess with an anechoic necrotic center and peripheral enhancement, as demonstrated by the CT examination. The basic US scan $\mathbf{b}$ does not allow complete visualization of the pathology.

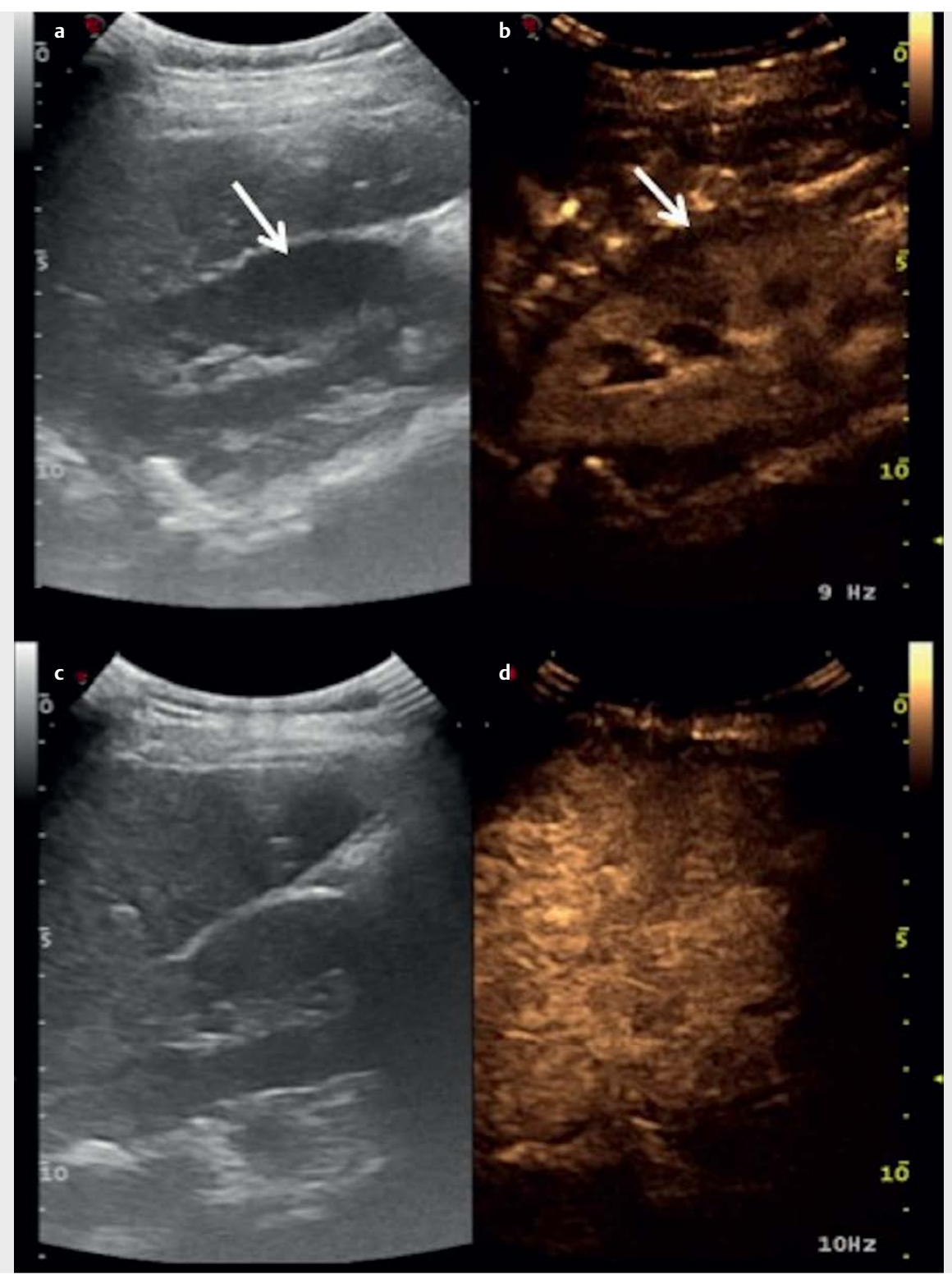

- Fig. 12 Acute pyelonephritis. The basic US image in a highlights a blurred hypoechoic area in the right kidney. After the administration of contrast medium, the CEUS examination $\mathbf{b}$ confirms the presence of a triangular area with reduced enhancement compared to the remaining parenchyma: the finding refers to an area of pyelonephritis (arrows). It is also associated with perirenal free fluid collection. The images in $\mathbf{c}$ and $\mathbf{d}$ are images from a follow up CEUS study after twenty days of antibiotic therapy. 
occasionally with internal septa with perifocal hyperemia can be easily demonstrated, especially during the arterial phase ( $>$ Fig. 11).

Similarly, in patients with acute pyelonephritis, the inflammatory areas within the cortical renal spaces are better depicted with CEUS than baseline US. These areas appear as rounded or wedgeshaped hypoenhancing foci within the normally enhancing renal parenchyma, with marginal hyperemia ( $\mathbf{F i g . ~ 1 2 ) ~ [ 6 2 ] . ~ T h e ~ p r e s - ~}$ ence of some perfusion can differentiate acute renal infection from infarction. In the latter scenario, the sonographic contrast signal is absent $[63,64]$.

\section{Limits of CEUS}

In an emergency setting, CEUS exam has several limitations, primarily related to the intrinsic limitations of US, i. e., intestinal movement, obesity, presence of free intraperitoneal air, and the inability of the patient to maintain breath-hold. Moreover, it does not have panoramic view, unlike CT which allows the complete visualization of the whole abdomen. CEUS may not diagnose deep or localized bleeding in a poorly studied abdominal parenchyma, such as the lower pole of the spleen or in the case of retroperitoneal collections. In these cases, an abdominal CT scan should be performed urgently. Despite these limitations, CEUS also has several advantages as explained in this review, including its rapid availability in an emergency context, patients who are bedridden, in intensive care units, or who are difficult to transport to the radiology department for a CT scan.

In conclusion, use of CEUS in the acute non-traumatic setting is not yet widespread, but it should be, especially because it is a simple and safe imaging modality in the study of inflammatory, ischemic, and hemorrhagic pathologies of the abdomen. Furthermore, CEUS improves the accuracy of baseline US imaging, while limiting the use of $\mathrm{CT}$ and related radiation exposure, especially in younger patients.

\section{Funding}

The authors declare that they did not receive any funding for the publication of this article.

\section{Ethical Approval}

All procedures performed in studies involving human participants were in accordance with the ethical standards of the institutional and national research committee and with the 1964 Helsinki declaration and its later amendments or comparable ethical standards.

Formal consent is not required for this type of study.

This article does not contain any studies with animals by any of the authors.

\section{Conflict of Interest}

The authors declare that they have no conflict of interest related to the publication of this article.
References

[1] Stoker ], Van Randen A, Laméris W et al. Imaging patients with acute abdominal pain. Radiology 2009; 253: 31-46

[2] Choe J, Wortman JR, Micheals A et al. Beyond appendicitis: Ultrasound findings of acute bowel pathology. Emerg Radiol 2019; 26: 307-317

[3] Van Randen A, Laméris W, Van Es HW et al. A comparison of the accuracy of ultrasound and computed tomography in common diagnoses causing acute abdominal pain. Eur Radiol 2011; 21: 1535-1545

[4] Hunt D, Romero J. Contrast-enhanced ultrasound. Magn Reson Imaging Clin N Am 2017; 25: 725-736

[5] Farina R, Catalano O, Stavolo C et al. Emergency radiology. Radiol Med 2015; 120: 73-84

[6] Dietrich CF, Averkiou M, Nielsen MB et al. How to perform contrast-enhanced ultrasound (CEUS). Ultras Int Open 2017; 3: E2-E15

[7] Emmanuel AL, Meijer RI, van Poelgeest E et al. Contrast-enhanced ultrasound for quantification of tissue perfusion in humans. Microcirculation 2019, doi: $10.1111 /$ micc. 12588

[8] Piccolo CL, Trinci M, Pinto A et al. Role of contrast-enhanced ultrasound (CEUS) in the diagnosis and management of traumatic splenic injuries. J Ultrasound 2018; 21: 315-327

[9] O'Connor JP, Aboagye EO, Adams JE et al. Imaging biomarker roadmap for cancer studies. Nat Rev Clin Oncol 2017; 14: 169-186

[10] Tedesco G, Sarno A, Rizzo G et al. Clinical use of contrast-enhanced ultrasonography beyond the liver: A focus on renal, splenic and pancreatic applications. Ultrasonography 2019; 38: 278-288

[11] Claudon M, Dietrich CF, Choi BI et al. Guidelines and good clinical practice recommendations for contrast enhanced ultrasound (CEUS) in the liver - update 2012; a WFUMB-EFSUMB initiative cooperation with representatives of AFSUMB, AIUM, ASUM, FLAUS and ICUS. Ultrasound Med Biol 2013; 39: 187-210

[12] Sidhu PS, Cantisani V, Dietrich CF et al. The EFSUMB guidelines and recommendations for the clinical practice of contrast-enhanced ultrasound (CEUS) in non-hepatic applications: update 2017 (short version). Ultrashall Med 2018; 154-180

[13] Cogley JR, O'Connor SC, Houshyar R et al. Emergent pediatric US: What every radiologist should know. Radiographics 2012; 32: 651-665

[14] Schreiber-Dietrich DG, Cui XW, Piscaglia F et al. Contrast-enhanced ultrasound in pediatric patients: A real challenge. Z Gastroenterol 2014; 52: 1178-1184

[15] Menichini G, Sessa B, Trinci M et al. Accuracy of Contrast-Enhanced Ultrasound (CEUS) in the identification and characterization of traumatic solid organ lesions in children: A retrospective comparison with baseline-US and CE-MDCT. Radiol Med 2015; 120: 989-1001

[16] Sidhu PS, Cantisani V, Deganello A et al. Role of contrast-enhanced ultrasound (CEUS) in paediatric practice: An EFSUMB position statement. Ultraschall in Med 2017; 38: 33-43

[17] Trinci M, Piccolo CL, Ferrari R et al. Contrast-enhanced ultrasound (CEUS) in pediatric blunt abdominal trauma. J Ultrasound 2019; 22: 27-40

[18] Pinto F, Valentino M, Romanini L et al. The role of CEUS in the assessment of hemodynamically stable patients with blunt abdominal trauma. Radiol Med 2015; 120: 3-11

[19] Miele V, Piccolo CL, Galluzzo M et al. Contrast-enhanced ultrasound (CEUS) in blunt abdominal trauma. Br J Radiol 2016; 89: 20150823

[20] Miele V, Piccolo CL, Sessa B et al. Comparison between MRI and CEUS in the follow-up of patients with blunt abdominal trauma managed conservatively. Radiol Med 2016; 121: 27-37 
[21] Tagliati C, Argalia G, Polonara G et al. Contrast-enhanced ultrasound in delayed splenic vascular injury and active extravasation diagnosis. Radiol Med 2019; 124: 170-175. doi:10.1007/s11547-018-0961-9

[22] Tagliati C, Argalia G, Graziani B et al. Contrast-enhanced ultrasound in the evaluation of splenic injury healing time and grade. Radiol Med 2019; 124: 163-169. doi:10.1007/s11547-018-0954-8

[23] Pinto F, Miele V, Scaglione M et al. The use of contrast-enhanced ultrasound in blunt abdominal trauma: advantages and limitations. Acta Radiol 2014; 55: 776-784

[24] Sessa B, Trinci M, lanniello $S$ et al. Blunt abdominal trauma: role of contrast-enhanced ultrasound (CEUS) in the detection and staging of abdominal traumatic lesions compared to US and CE-MDCT. Radiol Med 2015; 120: 180-189

[25] Mortele KJ, Cantisani V, Brown DL et al. Spontaneous intraperitoneal hemorrhage: Imaging features. Radiol Clin North Am 2003; 41: 1183-1201

[26] Catalano O, Sandomenico F, Mattace Raso M et al. Real-time, contrast-enhanced sonography: A new tool for detecting active bleeding. J Trauma 2005; 59: 933-939

[27] Martegani A, Aiani L, Borghi C. The use of contrast-enhanced ultrasound in large vessels. Eur Radiol 2004; 14: P73-P86

[28] Faccioli N, Foti G, Casagranda G et al. CEUS versus CT Angiography in the follow-up of abdominal aortic endoprostheses: Diagnostic accuracy and activity-based cost analysis. Radiol Med 2018; 123 : 904-909

[29] Corvino A, Sandomenico F, Setola SV et al. Added value of contrast-enhanced ultrasound (CEUS) with Sonovue ${ }^{\circledR}$ in the diagnosis of inferior epigastric artery pseudoaneurysm: report of a case and review of literature. J Ultrasound 2019; 22: 485-489. doi:10.1007/s40477-01900398-x

[30] Trinci M, Giangregorio C, Calabrese G et al. A rare case of non-traumatic intrasplenic pseudoaneurysms in a patient with acute T-cell lymphoblastic leukemia [published online ahead of print, 2019 Aug 1]. J Ultrasound 2019, 10.1007/s40477-019-00401-5. doi: doi:10.1007| s40477-019-00401-5

[31] Sugihara T, Koda M, Okamoto T et al. Two patterns of contrast-enhanced ultrasonography with Sonazoid in spontaneous rupture of hepatocellular carcinoma: A report of four cases. J Med Ultrason 2001; 45: 319-323

[32] Wells ML, Hansel SL, Bruining DH et al. CT for evaluation of acute gastrointestinal bleeding. Radiographics 2018; 38: 1089-1107

[33] Rosling M, Trenker C, Neessee A et al. Spontaneous and traumatic splenic rupture: Retrospective clinica, B-Mode and CEUS analysis in 62 patients. Ultrasound Int Open 2018; 4: E30-E34

[34] Dietrich CF, Averkiou M, Nielsen MB et al. How to perform Contrast-Enhanced Ultrasound (CEUS). Ultrasound Int Open 2018; 4: E2-E15

[35] Valentino M, Bertolotto M, Derchi L et al. The role of contrast enhanced ultrasound in acute scrotal diseases. Eur Radiol 2011; 21: 1831-1840

[36] Cantisani V, Bertolotto M, Weskott HP et al. Growing indications for CEUS: The kidney, testis, lymph nodes, thyroid, prostate, and small bowel. Eur J Radiol 2015; 84: 1675-1684. DOI: 10.1016/j. ejrad.2015.05.008

[37] Setola SV, Catalano O, Sandomenico F et al. Contrast-enhanced sonography of the kidney. Abdom Imaging 2007; 32: 21-28

[38] Von Herbay A, Schick D, Horger M et al. Low-MI-sonography with the contrast-agent SonoVue in the diagnosis of infarction of the spleen, kidney, liver and pancreas. Ultraschall in Med 2006; 27: 445-450

[39] Badea R, Lucan C, Suciu M et al. Contrast enhanced harmonic ultrasonography for the evaluation of acute scrotal pathology. Pictoril essay. Med Ultrason 2016; 18: 110-115
[40] Moschouris H, Stamatiou K, Lampropoulou E et al. Imaging of the acute scrotum: is there a place for contrast-enhanced ultrasonography? Int Braz J Urol 2009; 35: 692-705

[41] Trinci M, lanniello S, Galluzzo M et al. A rare case of accessory spleen torsion in a child diagnosed by ultrasound (US) and contrast-enhanced ultrasound (CEUS). J Ultrasound 2019; 22: 99-102

[42] Giannetti A, Biscontri M, Randisi P et al. Contrast-enhanced sonography in the diagnosis of acute mesenteric ischemia: A case report. J Clin Ultrasound 2010; 38: 156-160

[43] Kanzaki T, Hata J, Imamura $\mathrm{H}$ et al. Contrast-enhanced ultrasonography with Sonazoid for the evaluation of bowel ischemia. J Med. Ultrasonics 2012; 39: 161-167

[44] Hata J, Kamada T, Haruma K et al. Evaluation of bowel ischemia with contrast-enhanced US: initial experience. Radiology 2005; 236: 712-715

[45] Calabrese E, Catalano O, Nunziata A et al. Bedside contrast-enhanced sonography (CEUS) of the critically ill patient. J Ultrasound Med 2014; 33: 1685-1693

[46] Adamietz B, Wenkel E, Uder M et al. Contrast enhanced sonography of the gallbladder: A tool in the diagnosis of cholecystitis? Eur J Radiol 2007; 61: 262-266

[47] Sangrini E, Pecorelli A, Pettinari I et al. Contrast-enhanced ultrasonography to diagnose complicated acute cholecystitis. Intern Emerg Med 2015, doi:10.1007/s11739-015-1263-z

[48] Yashima YTT, Nakahara S, Ishida K et al. Contrast-enhanced ultrasonographic image of gangrenous gallbladder. J Med Ultrason 2011; 38: 239-241

[49] Incesu L, Yazicioglu AK, Selcuk MB et al. Contrast-enhanced power Doppler US in the diagnosis of acute appendicitis. Eur J Radiol 2004; 50: 201-209

[50] Gorg C, Egbring J, Bert T. Contrast-enhanced ultrasound of epiploic appendagitis. Ultraschall in Med 2009; 30: 163-167

[51] Golea A, Badea R, Socaciu M et al. Quantitative analysis of tissue perfusion using contrast-enhanced transabdominal ultrasound (CEUS) in the evaluation of the severity of acute pancreatitis. Med Ultrason 2010; 12: 198-204

[52] Addeo G, Beccani D, Cozzi D et al. Groove pancreatitis: A challenging imaging diagnosis. Gland Surg 2019; 8: S178-S187

[53] Ripollès T, Martinez-Pèrez MJ, Paredes JM et al. Contrast-enhanced ultrasound in the differentiation between phlegmon and abscess in Crohn's disease and other abdominal conditions. Eu J Radiol 2013; 82: e525-e531

[54] Buckley O, Geoghegan T, O'Riordan DS et al. Computed tomography in the imaging of colonic diverticulitis. Clin Radiol 2004; 59: 977-983

[55] Sessa B, Galluzzo M, Ianniello $S$ et al. Acute perforated diverticulitis: Assessment with multidetector computed tomography. Seminars Ultrasound CT MRI 2016; 37: 37-48. doi: 10.1053/j.sult.2015.10.003

[56] Esteban JM, Aleixandre A, Hurtado M] et al. Contrast-enhanced power Doppler ultrasound in the diagnosis and follow up of inflammatory abdominal masses in Crohn's disease. Eur J Gastroenterol Hepatol 2003; 15: 253-259

[57] Medellin A, Merrill C, Wilson SR. Role of contrast-enhanced ultrasound in evaluation of the bowel. Abdom Radiol 2017, doi:10.1007| s00261-017-1399-6

[58] Cozzi D, Moroni C, Addeo G et al. Radiological patterns of lung involvement in inflammatory bowel disease. Gastroenterol Res Pract 2018, doi:10.1155/2018/5697846

[59] Girlich C, Schacherer D, Lamby P et al. Innovations in contrast enhanced high resolution ultrasound improve sonographic imaging of the intestine. Clin Hemor Microcirc 2010; 45: 207-215 
[60] Catalano O, Sandomenico F, Mattace Raso M et al. Low mechanical index contrast-enhanced sonographic findings of pyogenic hepatic abscesses. AJR 2004; 182: 447-450

[61] Kim KW, Choi BI, Park Sh et al. Pyogenic hepatic abscesses: distinctive features for hypovascular hepatic malignancy on contrast-enhanced ultrasound with SH U 508A: early experience. Ultrasound Med Biol 2004; 30: 725-733

[62] Mitterberger M, Pinggera GM, Colleselli D et al. Acute pyelonephritis: comparison of diagnosis with computed tomography and contrast-enhanced ultrasonography. BJU Int 2008; 101: 341-344
[63] Fontanilla T, Minaya J, Cortès C et al. Acute complicate pyelonephritis: contrast enhanced ultrasound. Abdom Imaging 2012; 37: 639-346

[64] Bertelli E, Mercatelli L, Savi E et al. Surgical margin follow-up after nephron-sparing surgery: the possible role of CEUS.. J Ultrasound 2019, 10.1007/s40477-019-00413-1. doi: doi:10.1007/s40477-01900413-1 